\title{
Experimental study on the spray characteristics of a dual-manifold liquid-centered swirl coaxial injector
}

\author{
Ingyu Lee*, Jungsoo Yoon**, Gujeong Park*** and Youngbin Yoon****
}

School of Mechanical and Aerospace Engineering, Seoul National University, Seoul, Korea

\begin{abstract}
A throttleable rocket engine enables operational possibilities such as the docking of spacecraft, maneuvering in a certain orbit and landing on a planet's surface, altitude control, and entrance to atmosphere-less planets. Thus, throttling methods have long been researched. However, dual-manifold injectors, which represent one throttling method, have been investigated less than others. In this study, dual-manifold and single-manifold injectors were compared to determine the characteristics of dual-manifold injectors. Also, the effects of gas injection were investigated with various F/O ratios. To investigate the characteristics, mass flow rate, spray pattern, spray angle, and droplet size were measured. The spray angle and droplet size were captured by indirect photography. About 30 images were taken to assess the spray patterns and spray angle. Also, 700 images were analyzed to understand the droplet distribution and targeting area, moving to the right from the centerline with $1.11-\mathrm{cm}$ intervals. The droplet size was obtained from an image processing procedure. From the results, the spray angle showed two transition regions, due to swirl momentum in the swirl chamber regardless of the F/O ratio. The droplet size showed similar trends in both dual-manifold and single-manifold injectors except in the low mass flow rate region. In the case of the dual- manifold injector, the spray cone was not fully developed in the low mass flow rate region due to low angular momentum in the swirl chamber.
\end{abstract}

Key words: throttleable injector, dual manifold, coaxial injector, Sauter mean diameter (SMD), spray angle

\section{Introduction}

Space exploration technology could represent a national science technology. The lunar surface is a space exploration target still explored by only a few countries. For example, the United States sent a robot and humans in the Apollo project. To achieve lunar exploration, a modification in liquid propellant rocket engines, called throttling, is needed, as shown in Fig. 1 [1]. Throttling also enables operational possibilities: for example, docking of a spacecraft, maneuvering in a certain orbit, landing on the surface of planet, attitude control, and entrance to an atmosphereless planet. Thrust can simply be represented in followed equation.

$$
\mathrm{F}=\dot{m} \cdot v_{e}+A_{e}\left(p_{e}-p_{a}\right)
$$

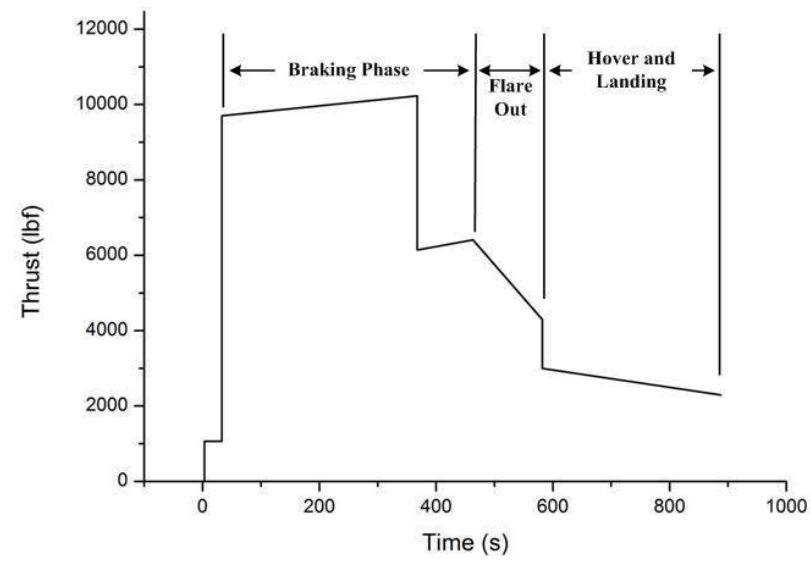

Fig. 1. Lunar module decent engine (LMDE) duty cycle
This is an Open Access article distributed under the terms of the Creative Commons Attribution Non-Commercial License (http://creativecommons.org/licenses/by$\mathrm{nc} / 3.0 /$ which permits unrestricted non-commercial use, distribution, and reproduction in any medium, provided the original work is properly cited.

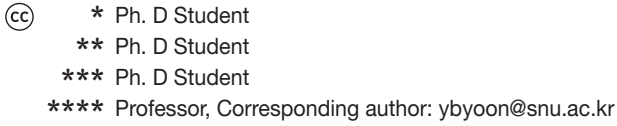


From this equation, it can be seen that the mass flow rate $(\dot{m})$, velocity at exit nozzle $\left(v_{e}\right)$, exit nozzle area $\left(A_{e}\right)$, and pressure difference $\left(p_{e}-p_{a}\right)$ influence the thrust. Thus, thrust can be modulated by controlling these variables.

Particularly for the mass flow rate modulation method, there are some variables that can be controlled, as follows:

$$
\dot{m}=C_{d} A_{e} \sqrt{2 \rho \Delta P}
$$

where $C_{d}$ is the discharge coefficient, and $\rho$ and $\Delta \mathrm{P}$ are the density of propellant and pressure drop, respectively. Mass flow rate modulation methods are classified according to these variables. The controlling exit nozzle area is generally called a pintle injector, and thrust is easy to predict because it is proportional to area variation. However, the method has the disadvantage that moving parts are positioned in extreme conditions, so durable materials are required.

Density modulation methods do not require moving parts, but can lead to instability in liquid flow from bubbles in the flow and the dissipation of excessive saturated gas. Moreover, high pressure drop is a representative form of pressure drop modulation methods. The high pressure drop method has been investigated for a long time, so many related studies have been conducted. Additionally, this can be combined with other methods. However, it has limitations in terms of accurate control and instability problems.

The controlling discharge coefficient can be represented by geometric variation, and a representative injector is the dual-manifold-type injector. In this injector, the mass flow rate is controlled using different manifolds. This requires four independent volumes. However, it shows good atomization efficiency in a large mass flow rate region and does not require moving parts. Thus, in the present study, this method was used for throttling [2].

Current rocket engines use liquid fuel and liquid oxidizer, liquid fuel and gaseous oxidizer, or gaseous fuel and liquid oxidizer as the propellant. Thus, many types of injector, such as the pintle injector, impinging injector, and coaxial injector, have been investigated in achieving high mixing efficiency, which, in turn, influences combustion efficiency. Coaxial injectors provide high atomization quality and stable operation over a wide thrust range. They can be classified according to the propellant phase or injection type. In terms of injection type, coaxial injectors are categorized as shear coaxial, liquid-centered swirl coaxial, and gas centered swirl coaxial injectors. The shear force between the central stream and annular stream supports breakup and mixing. The shear coaxial injector has been applied to the space shuttle main engine (SSME), the LE-7A engine, which generally has a central liquid jet and an annular gas jet [3] [4]. The breakup mechanism of swirl injector is caused by hydraulic instability on the liquid sheet. However, for a liquid-gas coaxial injector, the breakup mechanism is caused by the collision of kinetic energy between the gas flow and liquid sheet. A liquid-centered swirl coaxial injector with an annular jet was used in the RL-10 family and XLR-129 programs. Moreover, a gas-centered swirl coaxial injector with central jets was used in the RD-170 and other Russian rockets [5].

In this study, water and air were used for the oxidizer and fuel simulant in atmospheric conditions. The dualmanifold-type injector was used to modulate the liquid mass flow rate and to achieve high mixing efficiency, while an annular jet was used. Also, a single-manifold injector was designed to conduct a comparative study. The mass flow rate, spray pattern, and droplet size were measured to investigate the characteristics of the two systems.

\section{Experimental Apparatus and Methods}

\subsection{Experimental Apparatus}

As shown in Fig. 2, the injector was installed on a cold flow test rig. Two water lines were linked to each manifold to supply the simulant oxidizer, while a gas line was connected to the gas manifold to supply the simulant fuel. Both the water supply line and a static pressure sensor were linked on the top side of the top part, and the gas supply line and static sensor were linked on the side of the bottom part. Two pressurized water tanks were controlled independently to supply water to each manifold. Additionally, the air feed line was directly linked to the manifold from the gas tank and the mass flow meter (MFM, Alicat Scientific: M-1500SLPM-D) was positioned in

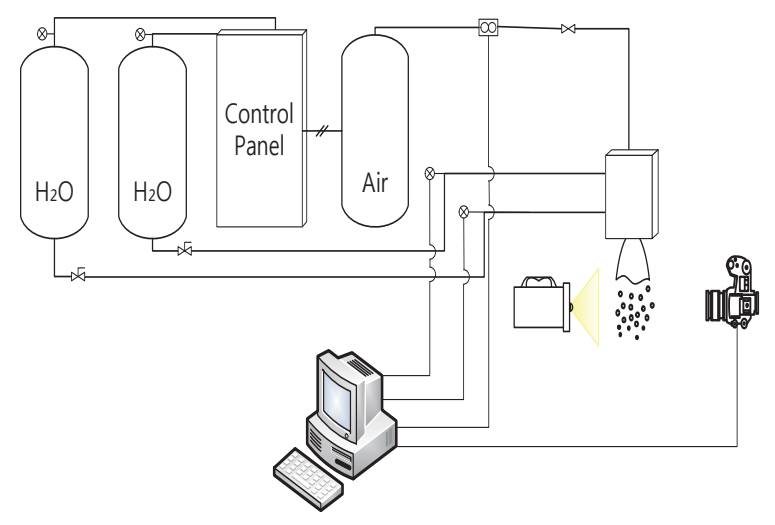

Fig. 2. Experimental apparatus 
front of the manifold to measure the mass flow rate in real time. The air mass flow rate was controlled by a manual regulator, in accordance with MFM data. The water mass flow rate was measured from the total mass flow rate at 10 $\mathrm{s}$ and $20 \mathrm{~s}$, and this was controlled in accordance with the manifold pressure.

As shown in Fig. 3 (a), for the liquid part, the top manifold had three tangential entries with diameter of $1.2 \mathrm{~mm}$ at every $120^{\circ}$, and the bottom manifold had two tangential entries with diameter of $1.0 \mathrm{~mm}$ at every $180^{\circ}$. The area ratio between tangential entry of the top manifold and bottom manifold was 2.16:1. Thus, the bottom manifold was used for low mass flow rate and the top manifold was used for mid mass flow rate, and both top and bottom manifolds were used for the large mass flow rate region. This ratio made it possible to modulate the mass flow rate. Fig. 3(b) illustrates the assembled injector. The barrier in the dualmanifold injector separated the manifold to modulate the mass flow rate. The manifolds operate individually as the liquid for each manifold is supplied from a different tank. Due to the system and injector aspect, the liquid mass flow rate can be regulated minutely.

Considering the gas part, the tangential entry area and nozzle were designed not to exceed the gas-line area. This is because the gas flow shows incompressible flow characteristics at the nozzle and tangential entry.

\subsection{Image Processing Method}

In terms of spray characteristics, droplet size could influence combustion efficiency [5]. Thus, this is an important parameter in injector design. To investigate droplet size, magnified images were obtained using a highresolution digital camera. The images were magnified through converters and an extension tube. Then, the obtained images were analyzed via post-processing procedures. An overview of the image processing method for measuring the droplets is given in Fig. 4.
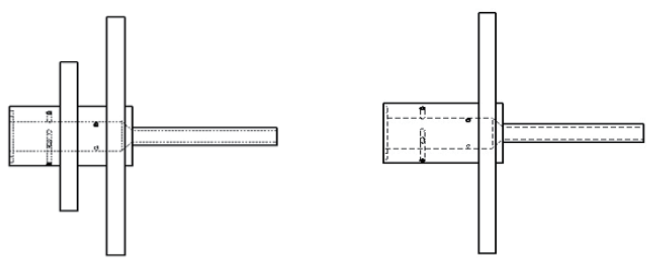

(a) Schematics of liquid and gas injector

Fig. 3. Schematic of injector
In the image-processing procedure, some undesired objects were detected that had to be removed. Additionally, drops that were out of focus or non-spherical needed to be filtered out. In the first step, the acquired original images were converted into raw images. Then, the images were converted into binary images where the gray level intensities between the droplets and background were distinguished at a threshold level determined as $50 \%$ gray level from the intersection point between the maximum gradient line and the $\mathrm{x}$-axis on a gray level histogram of the images [6]. This $50 \%$ gray level was an appropriate value for all the case to distinguish drops from background. Other values from 40 to $50 \%$ were assigned one-by-one and the drops could not be identified well. In the third step, the drop boundary was detected from the same intensity levels of adjacent pixels on each side of the object pixel. Then, the boundaries of the droplets were identified. In

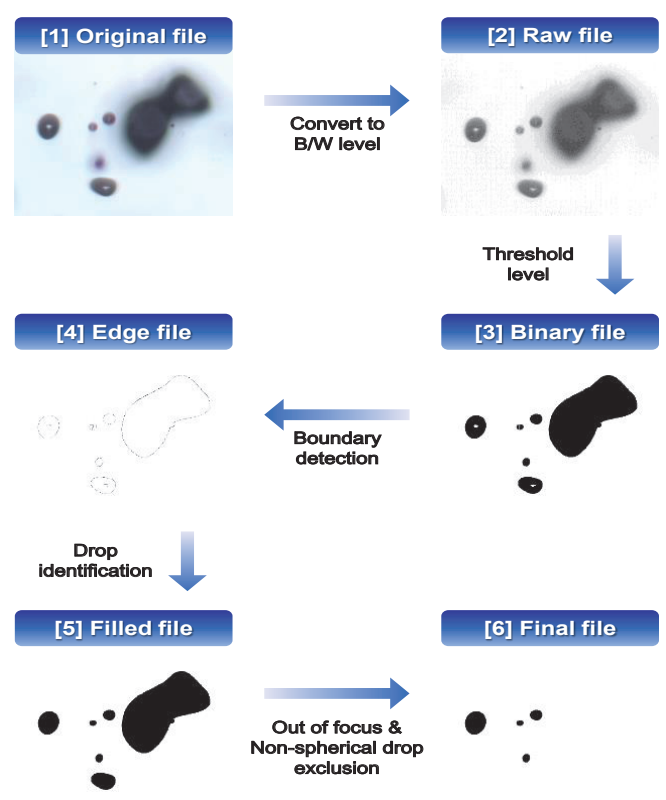

Fig. 4. Procedures of image processing method for drop size measurement

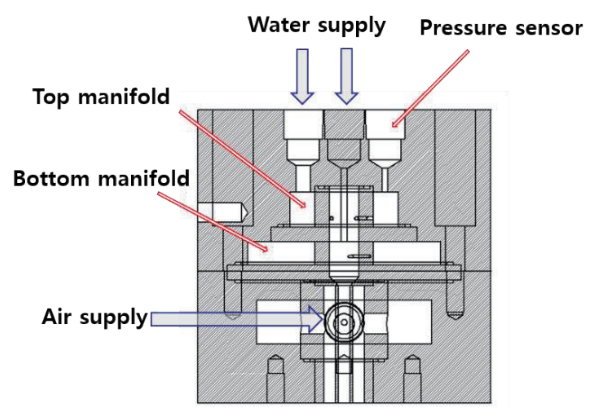

(b) Sectional view of injector assembly 
this step, unconnected boundaries such as drops that were partially out of the image frame were excluded. Then, the detected boundaries with closed curves were filled. Finally, some drops were excluded, which were nonspherical or out of focus. Thus, only appropriate droplets were evident in the final images for calculating the size of drops [5] [7].

To calculate the diameters of droplets, images with a spatial resolution of is $3.225 \mu \mathrm{m} /$ pixel were used. Moreover, 700 images were taken and averaged for each experimental case.

\subsection{Experimental Conditions}

In this study, the liquid manifold pressure and $\mathrm{F} / \mathrm{O}$ ratio were used as variables. The liquid mass flow rate was influenced mainly by the manifold pressure [8]. The pressure in the liquid varied from 1 to 8 bar because of limitations on material solidity. The other variable, the $\mathrm{F} / \mathrm{O}$ ratio, involved four cases $(0,1 / 10,1 / 8,1 / 6)$. While the $\mathrm{F} / \mathrm{O}$ ratio was fixed, the mass flow rate of the injected gas varied with an increase in the liquid mass flow rate.

\section{Result and Discussion}

\subsection{Mass Flow Rate}

The liquid mass flow rate was measured for each injector with varying pressure, as shown in Fig. 5(a). To measure the mass flow rate, injected water was stored for $20 \mathrm{~s}$ and this procedures were repeated three times. An averaged value was used for the mass flow rate. The dual-manifold injector had three types of mass flow rate at the same manifold pressure. Mass flow rate in the bottom manifold was lower

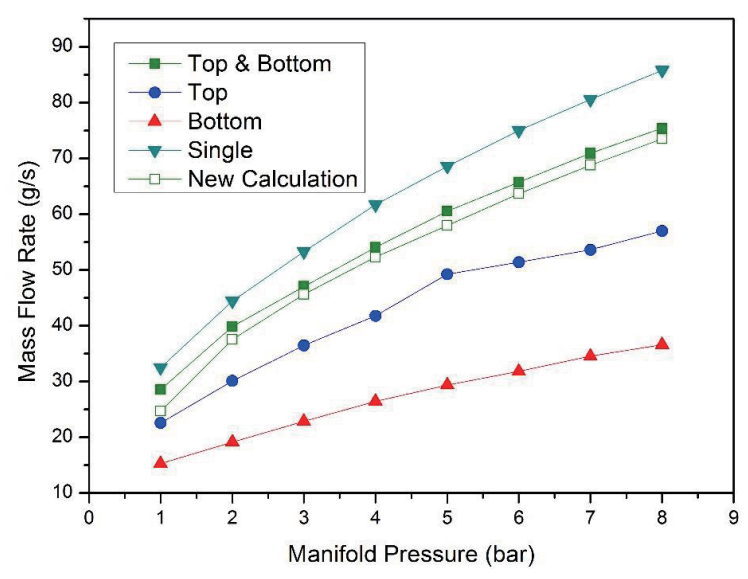

(a) Liquid mass flow rate than in the top manifold. This is because the tangential entry area was smaller in the bottom manifold. The area ratio between the top manifold and bottom manifold was 2.16. For the dual-manifold injector, the range for the mass flow rate was $20.25 \sim 100 \%(15.27 \sim 75.40 \mathrm{~g} / \mathrm{s})$, while that for the single-manifold injector was 37.8 100\% (32.47 85.78 g/s). These percentages were determined by dividing the mass flow rate by maximum mass flow in each injectors. The gas mass flow rate was measured with real-time measurements and controlled by a manual regulator, while the liquid mass flow rate varied and the $\mathrm{F} / \mathrm{O}$ ratio was set at $0,1 / 10,1 / 8$, or 1/6. The results are shown in Fig. 5(b).

When one manifold was pressurized, the other was also pressurized, as illustrated in Fig. 6 [9]. The target manifold means the actually pressurized manifold and other manifold is pressurized incidentally. It was intended to calculate the mass flow rate while the top and bottom manifolds were used and measured how the manifold pressure affects the other manifold. Also, when using both manifolds, the mass flow rates could be predicted from the results of pressure data from a single manifold injection in the dual-manifold injector. The mass flow rate prediction was based on the pressure difference between the target manifold and the other manifold. The simple relationship between mass flow rate and pressure difference is as follows. This equation is generally used in jet injection calculations:

$$
\dot{m}=C_{d} A_{e} \sqrt{2 \rho \Delta P}
$$

The pressure difference $(\Delta P)$, density $(\rho)$, tangential entry area $\left(A_{e}\right)$ and, mass flow rate $(\dot{m})$ are already known. The discharge coefficient $\left(C_{d}\right)$ can be calculated and it was 0.45 and 0.46 . Using these discharge coefficients, the mass flow rates through both manifolds were calculated and

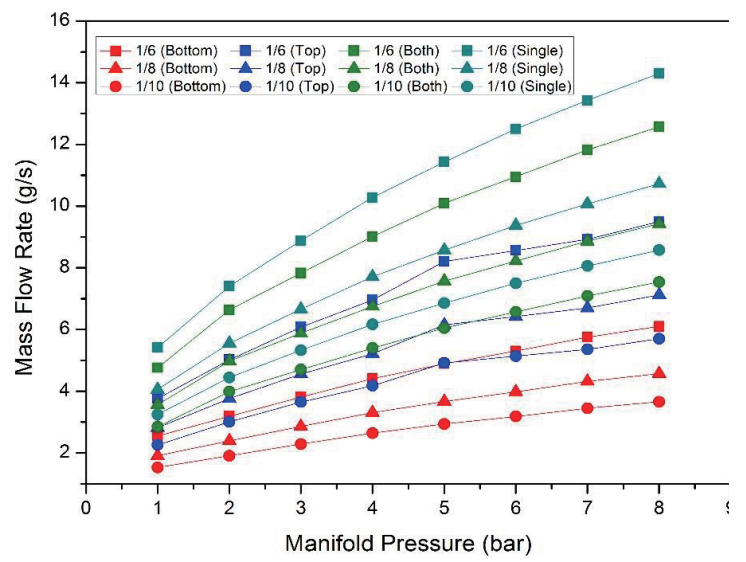

(b) Gas mass flow rate

Fig. 5. Mass flow rate via injection pressure 


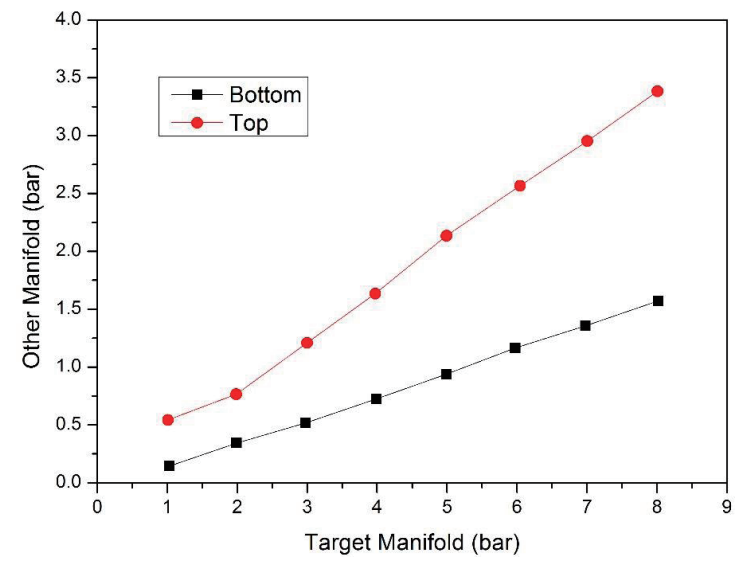

Fig. 6. Pressure relationships between the target manifold and the other manifold.

illustrated in Fig. 5 as "New Calculation." In a multi-low swirl injection process, there is no appropriate prediction methods. So, it is not the same, but this procedure is worthy for predicting the mass flow rate.

\subsection{Spray Pattern}

The spray angle is a significant parameter in combustion systems using swirl injectors. It influences the droplet distribution, affecting the ignition and arrangement of multi-elements injectors [9]. Fig. 7 illustrates the spray shapes of the dual-manifold and single-manifold injectors. Moreover, Fig. 7(b) shows the spray shape without gas injection. This study is aimed to design the bi-propellant injector, not a mono-propellant injector. Thus, this does not demonstrate the actual condition, but can instead be used for comparison. The condition in Fig. 7(a) is similar to the actual operation that illustrates the spray shape with gas injection $(\mathrm{F} / \mathrm{O}$ ratio $=1 / 6)$ and it is arranged in mass flow rate order. To control the mass flow rate, the

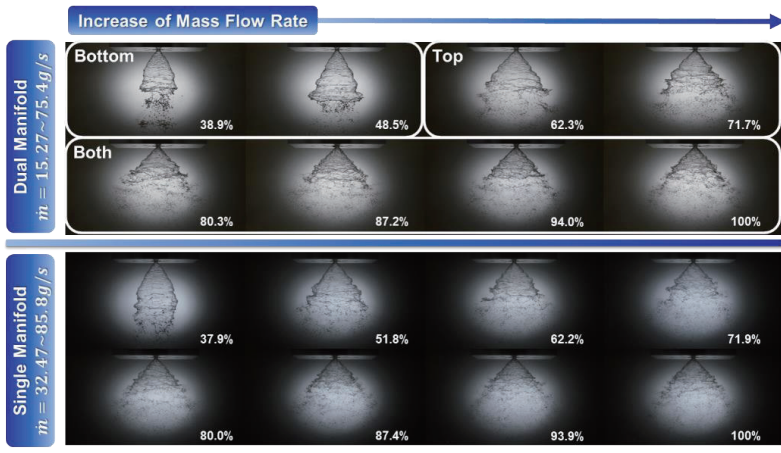

(a) Injection without gas injection top manifold and bottom manifold were modulated independently. This means that for the low mass flow rate region (20.25 45.79\%), the bottom manifold was used. For the mid (48.35 71.08\%) and high mass flow rate regions (71.08 100\%), the top manifold and both manifolds were used for each region. Regardless of gas injection, the spray angle was increased with an increase in mass flow rate for the dual- and single-manifold injectors. In the case of the low mass flow rate region, the spray cone was not fully developed.

For a detailed description of the spray angle, it was quantified by summation of the left half-spray angle and the right half-spray angle. The half-spray angle is measured from the centerline to the left or the right side of the spray sheet. In addition, 30 images were taken and averaged for this one experimental case to obtain an accurate value. The spray angle via the mass flow rate is shown in Fig. 8. For both the dual-manifold and single single-manifold injectors, the spray angle shows showed a tendency to increase with the mass flow rate through a certain manifold. Spray angle transition occurred when the manifold changed. To describe this transition in detail, the angular momentum in the swirl chamber is can be introduced. The initial angular momentum in swirl chamber can be expressed as:

$$
\dot{m} u R=\frac{\dot{m}^{2}}{\rho A} R=\frac{4 \dot{m}^{2}}{\rho n_{T} \pi d_{p}^{2}} R
$$

The first transition is simple to analyze. The mass flow rate from bottom manifold was relatively lower than the mass flow rate from the top manifold. Thus, the swirl momentum in the swirl chamber was relatively low. The second transition was caused by interaction between the top manifold injection and bottom manifold injection. As shown in Fig. 9, the angular momentum in the swirl chamber was calculated from the initial angular
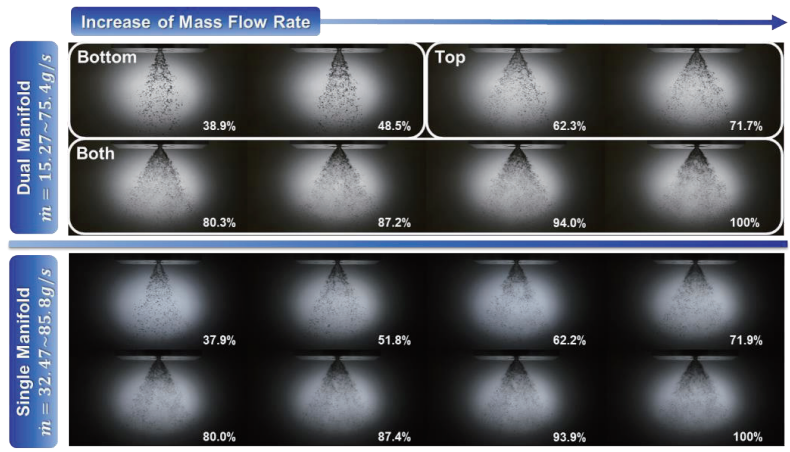

(b) Injection with gas injection

Fig. 7. Spray pattern at various mass flow rate 


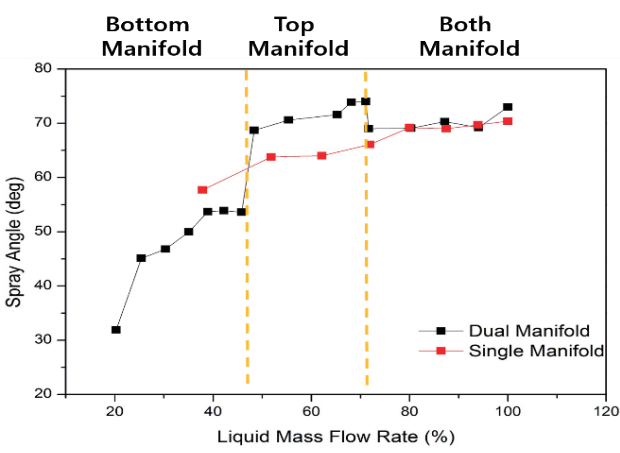

(a) $\mathrm{F} / \mathrm{O}$ ratio $=0$

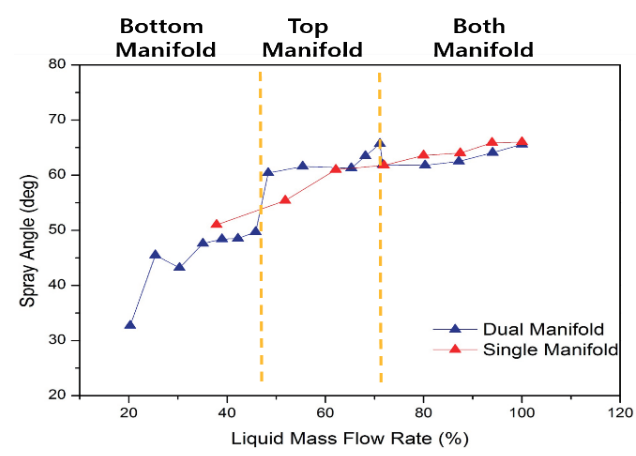

(c) $\mathrm{F} / \mathrm{O}$ ratio $=1 / 8$

Fig. 8. Spray angle via mass flow rate

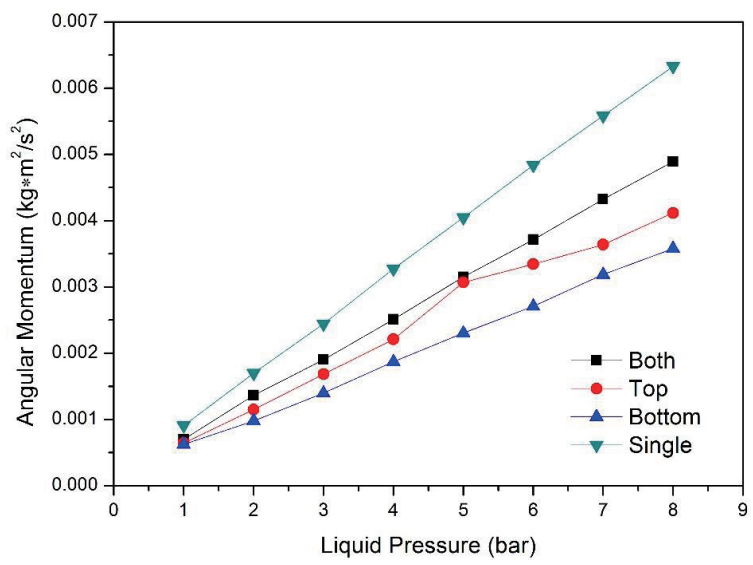

Fig. 9. Angular momentum in swirl chamber

momentum equation and the mass flow rate prediction in the last section. When using both manifolds, the angular momentum from the bottom manifold was lower than that from the top manifold. Thus, when the lower angular momentum collided with the higher angular momentum, the higher momentum decreased. As a result of this collision, the angular momentum in the high mass flow rate range can be lower than that of the mid-range.

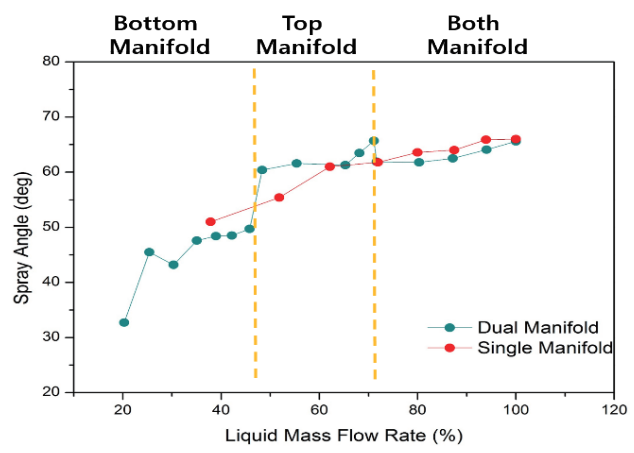

(b) $\mathrm{F} / \mathrm{O}$ ratio $=1 / 10$

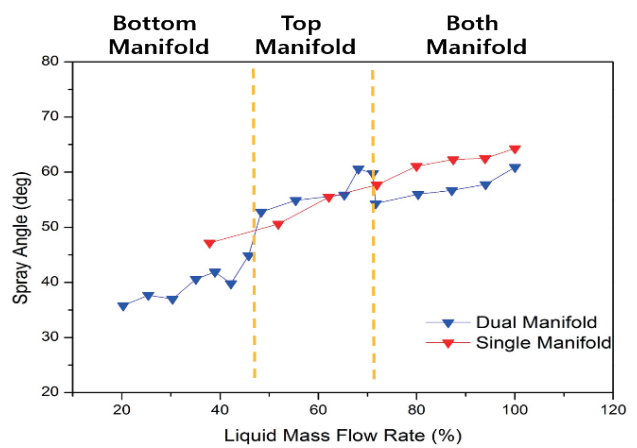

(d) $F / O$ ratio $=1 / 6$

\subsection{Droplet Distributions}

Droplet size is an important parameter in the combustion process. This parameter represents the atomization quality because fine atomization facilitates rapid vaporization and better mixing efficiency. Most previous studies on spray characteristics have focused on predicting drop size and distribution [10]. This is because these parameters have been shown to strongly affect the performance, stability limits, and pollutant emissions. To observe the atomization characteristics of this injector, the drop size was measured using the image processing method mentioned in Section 2.2.

To analyze the drop size distribution, some variation was introduced. First, to measure the drop size, the Sauter mean diameter (SMD) was used. The SMD is generally used in mass transfer and reaction fields [10]:

$$
\operatorname{SMD}\left(D_{32}\right)=\frac{\sum N_{i} D_{i}^{3}}{\sum N_{i} D_{i}^{2}}
$$

Moreover, the non-dimensional radial distance (D/W) was used, which is the radial distance from the center-line divided by the half-spray width. This non-dimensional radial distance helps to analyze the SMD distribution related to liquid sheet width: 


$$
\begin{aligned}
& \text { Half Spray Width }=10 \times \tan \frac{\alpha}{2} \\
& D / W=\frac{\text { Radial Distance }}{\text { Half Spray width }}
\end{aligned}
$$

The spray angle $(\alpha)$ was measured in the previous section; this was used for non-dimensional radial distance.

An image was taken where the horizontal distance was 10 $\mathrm{cm}(\mathrm{L} / \mathrm{D}=17)$ from the exit of the nozzle. At this position, the spray was almost broken. Furthermore, the radial point was varied from the center line to $8.88 \mathrm{~cm}$ at intervals of $1.11 \mathrm{~cm}$; this is why the magnified image frame size is $1.11 \mathrm{~cm}$.

Figure 10 shows the SMD distribution for the dualmanifold injector with various liquid mass flow rates. Each graph shows the SMD with the F/O ratio difference. For all $\mathrm{F} / \mathrm{O}$ ratio cases, the SMD using the bottom manifold is higher than the others and most drops are observed within the spray width. Because the angular momentum was too small, the injected liquid merged again somewhere downstream. Otherwise, the SMDs using the top manifold

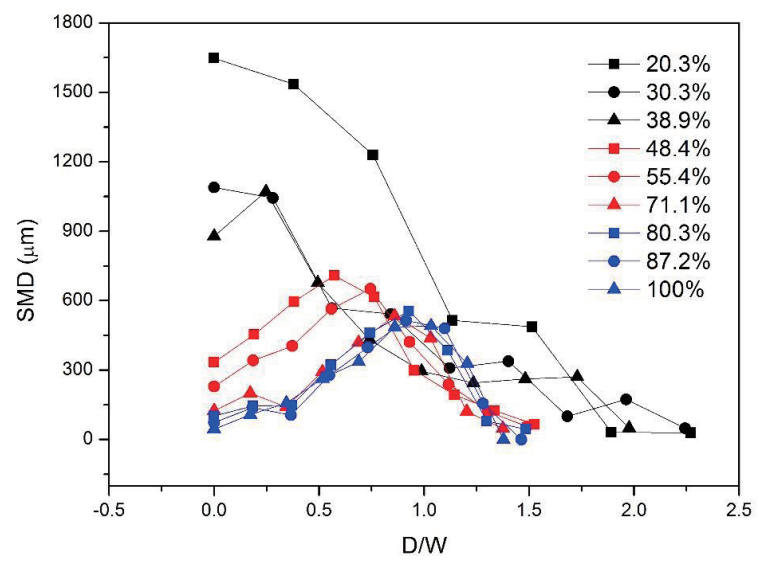

(a) $\mathrm{F} / \mathrm{O}$ ratio $=1 / 10$ and both manifolds where the mass flow rate was over $48.4 \%$ had similar aspects. The droplet sizes were similar because the spray angle values were also relatively similar. The SMD increased until near $\mathrm{D} / \mathrm{W}=1$ and then decreased. This means that the droplets near the spray sheet were the biggest.

From the perspective of $\mathrm{F} / \mathrm{O}$ ratio variation, the maximum SMD radial position moved slightly to the right with an increase in the $\mathrm{F} / \mathrm{O}$ ratio in the mid-mass flow rate region. The droplet size was largest at about $\mathrm{D} / \mathrm{W}=0.7$, where the $\mathrm{F} / \mathrm{O}$ ratio $=1 / 10$; when the $\mathrm{F} / \mathrm{O}$ ratio was $1 / 6$, the maximum droplet $\mathrm{D} / \mathrm{W}$ was 1 . Relatively similar results were seen for the high mass flow rate region. However, the region where both manifolds were used showed similar results regardless of variation in the F/O ratio. From this result, it was found that an increase in the $\mathrm{F} / \mathrm{O}$ ratio allows simple prediction of the maximum SMD position in the spray. Additionally, regardless of $\mathrm{F} / \mathrm{O}$ ratio variation, substantial change in SMD was obtained. However, from the perspective of the mean SMD, the droplet sizes are slightly different.

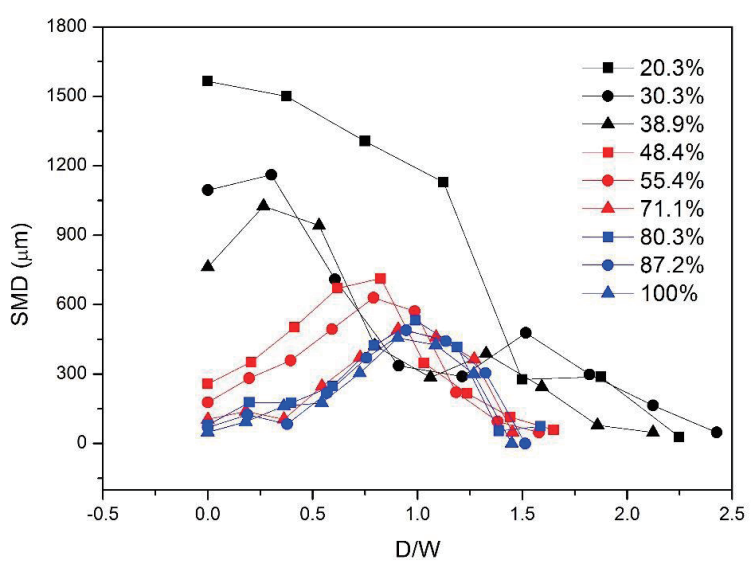

(b) $F / O$ ratio $=1 / 8$

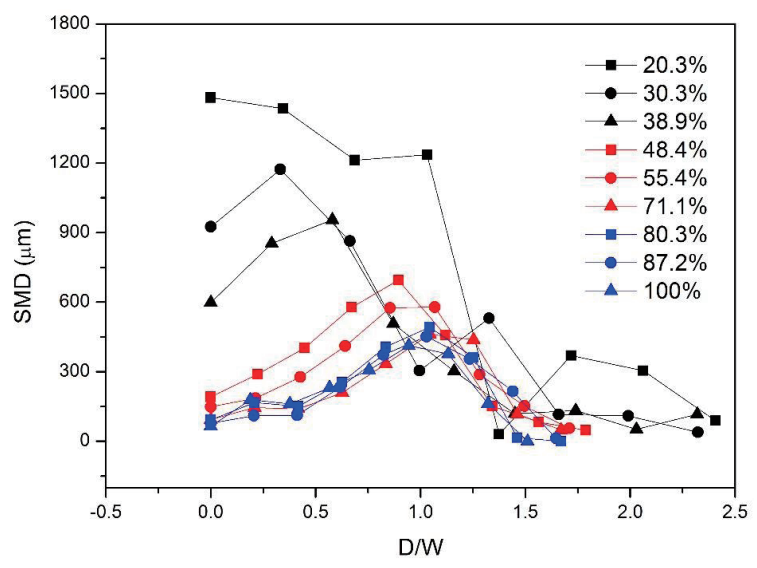

(c) $\mathrm{F} / \mathrm{O}$ ratio $=1 / 6$

Fig. 10. SMD distribution for dual-manifold injector 
This phenomenon is due to an increase in gas momentum, which is the dominant mechanism in the breakup. A detailed description will be discussed in the SMD part.

Distinct from the SMD result for the dual-manifold injector, the SMD distribution for the single-manifold injector is shown in Fig. 11. In the single-manifold injector, all the SMD distributions were similar; that is, the nondimensional radial distance of the maximum SMD was 0.75 0.90 , regardless of the $\mathrm{F} / \mathrm{O}$ ratio. As an exception, the position where the mass flow rate was $37.9 \%$ was lower, and the SMD value was higher, for the same reason as seen in the low mass flow rate range with the dual-manifold injector.

From Fig. 10 and 11, the aspects of droplet size and distribution were similar at mass flow rates higher than $38.9 \%$. This results from the geometric characteristics. As mentioned earlier, the only difference between single- and dual-manifold injector is a barrier separating the manifolds. Thus, almost similar aspects are seen over all mass flow rate conditions. However, the dual-manifold injector has benefits on mass flow range with the same pressure range.

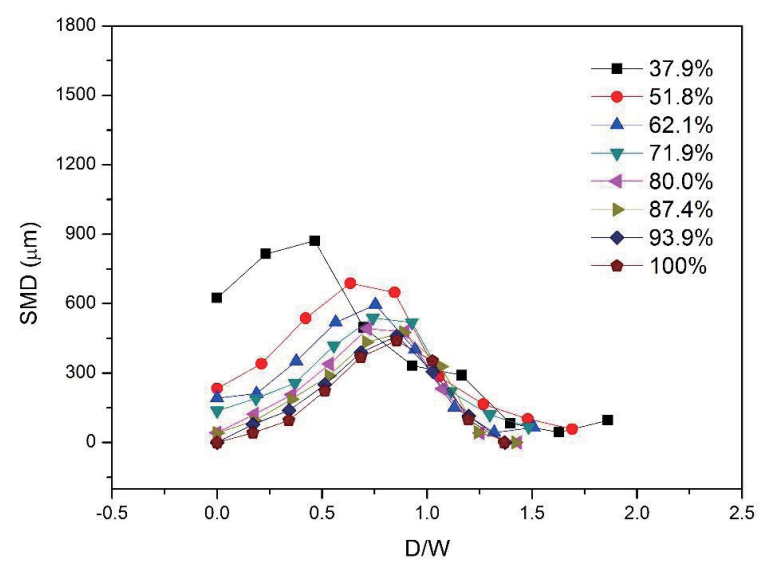

(a) $\mathrm{F} / \mathrm{O}$ ratio $=1 / 10$
One limitation of this image-processing method is that point data were used, so that the measured SMD information was only valid for a specific point. Thus, the concept of mean SMD is needs to be introduced to understand the effect of conditions. The following equation is a modified equation for $\left(\overline{D_{30}}\right)$, as suggested by Zaller and Klen [11], where (Data Rate) ${ }_{i}$ represents the number of droplets in this experience case:

$$
\overline{S M D}=\frac{\sum_{i}(\text { Data Rate })_{i}(\text { Area })_{i}\left(D_{32}\right)_{i}}{\sum_{i}(\text { Data Rate })_{i}(\text { Area })_{i}}
$$

The mean SMD was calculated to understand the effects of mass flow rate, radial position, and F/O ratio. Fig. 12(a) shows the mean SMD variation with an increase in the mass flow rate and this mean SMD is calculated from SMD in all radial position. The open symbols represent the single-manifold injector, while solid symbols represent the dual-manifold injector. All mean SMDs decreased with an increase in mass flow rate. The single-manifold injector

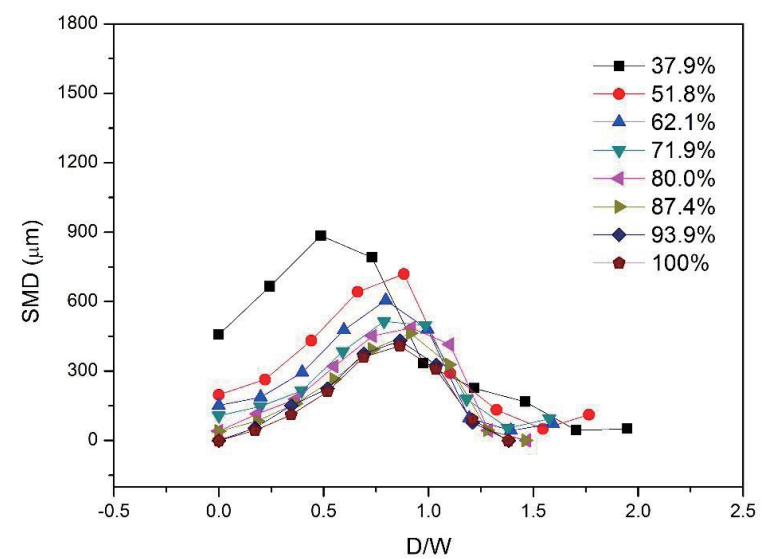

(b) $F / O$ ratio $=1 / 8$

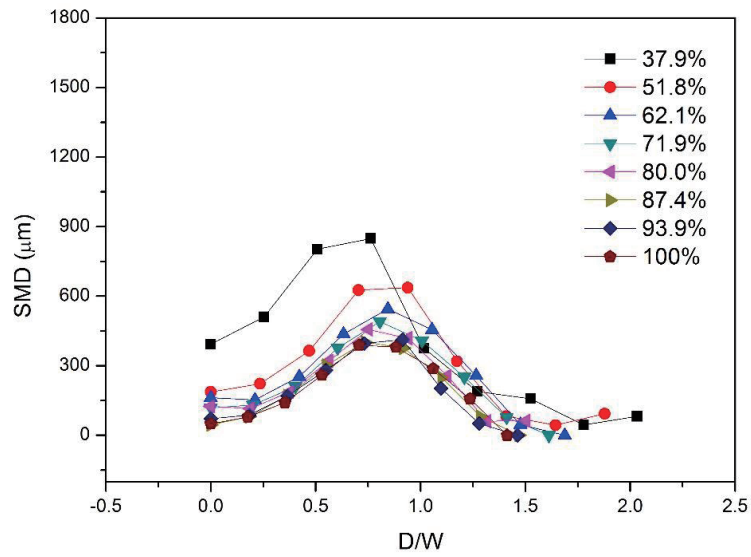

(c) $\mathrm{F} / \mathrm{O}$ ratio $=1 / 6$

Fig. 11. SMD distribution for single-manifold injector 
could not operate at deep throttling under $38 \%$; over $38 \%$ in the mass flow rate region, the mean SMD showed almost the same results. From the perspective of change in the $\mathrm{F} / \mathrm{O}$ ratio, the mean SMD showed a smaller change with the increase in $\mathrm{F} / \mathrm{O}$ ratio. The collision with the liquid sheet and air jet is the dominant mechanism in the breakup. As the air mass flow rate increases, the momentum in the air jet is increased. Thus, when $\mathrm{F} / \mathrm{O}$ ratio is increased, strong collisions occur. So, the droplet is well atomized. In the dual-manifold injector, where the $\mathrm{F} / \mathrm{O}$ ratio was $1 / 6$, the mean SMD was lowest in almost every range. However, for the single-manifold injector, the mean SMD with an F/O ratio $=0$ or $1 / 6$ was found to be lowest.

Figure 12(b) illustrates the mean SMD variation with an increase of $\mathrm{D} / \mathrm{W}$ and the mean SMD was calculated from the SMD for all mass flow rates. As shown in Fig. 12(b), mean droplet size decreased with distance from the centerline. In the case of the dual-manifold injector, the mean SMD decreased to a radial distance of $3.33 \mathrm{~cm}$. This is because the effect of injection through the bottom manifold was significant at this point. Moreover, at the same point, injection via the top manifold or both manifolds resulted in lower SMD. After the $3.33 \mathrm{~cm}$ point, the tendency of the mean SMD was found to be similar to the results for SMD distribution. For the singlemanifold injector, the mean SMD showed reverse results under the $3.33 \mathrm{~cm}$ range. This means that near the centerline, a large number of drops was distributed when the mass flow rate was $37.9 \%$. However, after this point, this injector had the same characteristics as the dual-manifold injector.

Without gas injection $(\mathrm{F} / \mathrm{O}$ ratio $=0)$ different tendencies were demonstrated in both injectors; that is, the diameter maintained a higher size. Additionally, the mean SMD was higher over the $7.77 \mathrm{~cm}$ region. This was driven by the relationships between the spray angle and gas momentum.

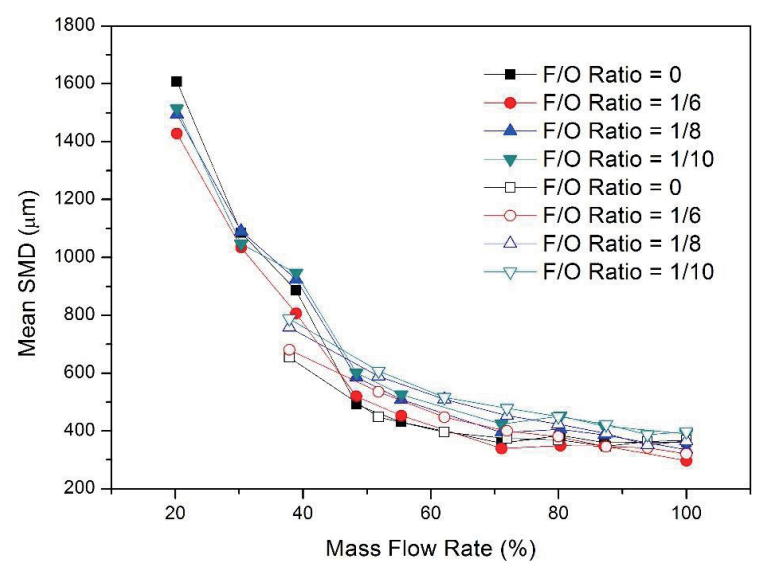

(a) Mean SMD via mass flow rate
In other cases, the break mechanism collides with the liquid sheet and gas, resulting in high atomization quality. However, without gas injection, the break mechanism only involves the swirl momentum and frictional forces.

\section{Conclusions}

When using a dual-manifold injector, spray angle variation $(\Delta \alpha)$, which could represent a range of spray angles and is a parameter of spray angle stabilization, decreases with an increase in $\mathrm{F} / \mathrm{O}$ ratio. In contrast, using a single-manifold injector, spray angle variation increases with an increase in the $\mathrm{F} / \mathrm{O}$ ratio.

During the throttling process, two transitions occur due to angular momentum in the swirl chamber. The first transition is caused by using different manifolds, while the second transition is caused by collisions between the higher angular momentum from the top manifold and the lower angular momentum from the bottom manifold.

For both injectors, the SMD increases under $\mathrm{D} / \mathrm{W}=$ 1.00 , and then decreases. That is, the diameter of droplets is maximal within the spray cone. However, at low mass flow conditions in both injectors, relatively large drops were observed. This is because the liquid had low swirl momentum; thus, this region needs to be improved.

The mean SMD via the mass flow rate decreases with an increase in the mass flow rate. Both injectors showed similar mean SMD information at a mass flow rate of over $40 \%$. The mean SMD via radial distance also decreased further from the centerline for the dual-manifold injector. In contrast, the mean SMD in the single-manifold injector increased up to $2.22 \mathrm{~cm}$ from the centerline; after that, it showed a similar tendency.

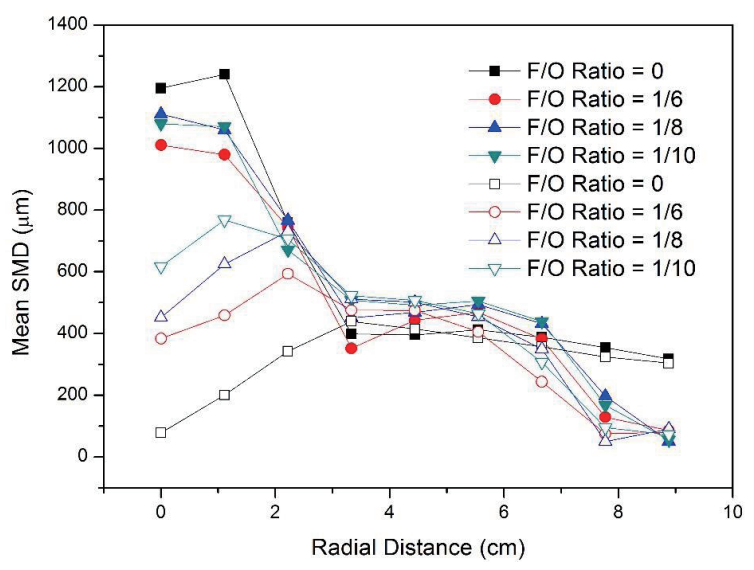

(b) Mean SMD via radial distance

Fig. 12. Mean SMD Distribution 


\section{Acknowledgements}

This workwas supported by NationalResearchFoundation Grant, funded by the Korean Government (NRF-2011220-D00023), and the Institute of Advanced Aerospace Technology at Seoul National University and Advanced Research Center Program (NRF-2013R1A5A1073861) through the National Research Foundation of Korea (NRF) grant, funded by the Korea government (MSIP), contracted through Advanced Space Propulsion Research Center at Seoul National University.

\section{References}

[1] G. A. Dressler, "Summary of Deep Throttling Rocket Engine with Emphasis on Apollo LMDE", 42nd AIAA/ASME/ SAE/ASEE Joint Propulsion Conference \& Exhibit, 9-12 July 2006.

[2] Matthew J. Casiano, James R. Hulka, and Vigor Yang, "Liquid-Propellant Rocket Engine Throttling: A Comprehensive Review", Journal of Propulsion and Power, Vol. 26, No. 5, September-October 2010.

[3] Y. Hardalupas and J. H. Whitelar, "Characteristics of
Sprays Produced by Coaxial Airblast Atomizers", Journal of Propulsion and Power, Vol. 10, No. 4, July-Aug 1994.

[4] J. C. Lasheras and E. J. Hopfinger, "Liquid Jet Instability and Atomization in A Coaxial Gas Stream", Annual Review of Fluid Mechanics, Vol. 32, 2000, pp. 275-308.

[5] J. H. Im, Spray Characteristics of Gas-Liquid Swirl Coaxial Injectors for Liquid Rocket Engines, Ph. D Thesis, 2010.

[6] J. Y. Kim, J. H. Chu and S. Y. Lee, "Improvement of Pattern Recognition Algorithm for Drop Size Measurement", Atomization and Sprays, Vol 9, 1999, pp.313-329.

[7] Y. J. Choo and B. S. Kang, "Measurement of Sizes and Velocities of Spray Droplets by Image Processing Method", ILASS-Korea, 2002, pp.121-128.

[8] V. G. Bazarov, "Throttleable Liquid Propellant Engines Swirl Injectors for Deep Smooth Thrust Variations", 30th AIAA/ASME/SAE/ASEE Joint Propulsion Conference, 27-29 June 1994.

[9] J. S. Yoon, Characteristics of Throttelabe Liquid Rocket Injector with Dual Manifold, MS Thesis, 2010

[10] Arthur H. Lefevbre, Atomization and Sprays, Hemisphere Publishing Corporation, 1989.

[11] M. Zaller and M. Klem, "Shear Coaxial Injector Spray Characteristics", Liquid Rocket Engine Combustion Instability, AIAA, pp.191-213. 